\title{
PICTORIAL ESSAY
}

\section{Imaging Features and Techniques in Assessment of Cleft Palate: a Pictorial Essay}

\author{
AWT Chin, KS Tse, HC Lee, YW Hon, KL Lo \\ Department of Radiology and Organ Imaging, United Christian Hospital, Hong Kong
}

\section{INTRODUCTION}

Cleft lip and palate deformities occur in around 1 in 600 live births in Hong Kong. ${ }^{1}$ Our institution has been offering a one-stop territory-wide integrated multidisciplinary service to manage patients with complicated cleft deformities within Hong Kong. ${ }^{2}$ The normal palate arises in utero from fusion of the primary and secondary palates and should occur at around 4 to 6 weeks gestation and 8 to 12 weeks gestation, respectively. Failure of fusion may cause cleft lip and / or cleft palate depending on the severity. ${ }^{3}$ Cleft palate may be one of the manifestations of clinical syndromes such as Pierre Robin sequence. In such patients, there will be additional faciocranial manifestations.

The levator veli palatini muscles help retract the velum (soft palate) to allow separation of the nasopharynx from the oropharynx by complete coverage of the soft palate against the posterior pharyngeal wall. Patients with cleft palate have abnormalities of the levator veli palatini muscle, resulting in suboptimal velopharyngeal closure, known as velopharyngeal insufficiency. This causes hypernasality that impairs speech development. In the past, nasoendoscopy and videofluoroscopy were used to assess velopharyngeal anatomy. However, nasoendoscopy is invasive as it involves placement of an endoscope into the pharyngeal region and may cause patient anxiety and discomfort, especially in children. ${ }^{4}$ In addition, videofluoroscopy, as with other fluoroscopic studies, has inherent measurement errors that may vary with patient positioning. ${ }^{4}$ With the advances in crosssectional imaging modalities including computed tomography (CT) and magnetic resonance imaging (MRI), non-invasive assessment of bony anatomy and velopharyngeal musculature with easily reproducible anatomy and measurements is now feasible. Preoperative imaging assessment assists paediatric and maxillofacial surgeons in surgical management.

In this pictorial essay, we describe technical considerations in imaging and highlight the relevant radiological anatomy and pathological features in CT and MRI in the assessment of patients with cleft palate.

\section{PATIENT PREPARATION}

We aim to perform CT and MRI studies, whenever possible, without sedation. The images used here have been obtained from patients without sedation. When

Correspondence: Dr AWT Chin, Department of Radiology and Organ Imaging United Christian Hospital, Kwun Tong, Hong Kong Email: anthonywtchin@gmail.com

Submitted: 20 Feb 2019; Accepted: 11 Apr 2019

Contributors: All authors contributed to the concept or design, acquisition of data, analysis or interpretation of data, drafting of the manuscript, and critical revision for important intellectual content. All authors had full access to the data, contributed to the study, approved the final version for publication, and take responsibility for its accuracy and integrity.

Conflicts of Interest: The authors have no conflicts of interest to disclose. All authors had full access to the data, contributed to the study, approved the final version for publication, and take responsibility for its accuracy and integrity.

Funding/Support: This pictorial essay received no specific grant from any funding agency in the public, commercial, or not-for-profit sectors.

Ethics Approval: Ethics approval and need for patient consent for this pictorial essay was waived by the ethics committee of the Hospital Authority, Hong Kong. 
performing imaging studies in children, it is crucial to strike a balance between the risk of motion artefacts that can occur in the absence of sedation and altered muscle tone that occurs with sedation. Currently, there is no available evidence to show how sedation affects cleft palate measurements. A recent study by Ali et $\mathrm{al}^{5}$ revealed that velopharyngeal dimensions obtained from static MRI correlate with speech data, and it included children who had been sedated. Uncooperative paediatric patients are given light sedation with oral chloral hydrate just before imaging to prevent motion artefacts. It is clearly stated in radiology reports if sedation has been given. In most of our cases we obtained CT and MRI images without patient sedation.

Before examination, patients were clearly instructed to breathe through their nose and remain at rest without speech or swallowing. Patients undergoing MRI wore an MR-compatible headset so radiographers could communicate with them. Cushions and foams were used to stabilise the patients in a supine position to minimise motion during scanning.

\section{COMPUTED TOMOGRAPHY}

Plain CT is usually performed for patients with complicated or syndromic cleft palate such as Pierre Robin sequence to better assess bony detail and facilitate three-dimensional (3D) printing for preoperative planning. Our centre uses a 64-detector single-source CT scanner (LightSpeed VCT; GE Healthcare, Chicago [IL], United States). Plain CT from the skull base to the entire mandible is performed. Helical mode is used during scanning. Depending on the patient's body habitus, CT studies in older children adopt $120 \mathrm{kVp}$, $164 \mathrm{mAs}$ and pitch of 0.984 . These images are obtained in axial acquisition at 5-mm thickness, and reformatted to $0.62-\mathrm{mm}$ slices. For young children and infants, CT studies adopt $80 \mathrm{kVp}, 125 \mathrm{mAs}$ and pitch of 0.968 . These images are obtained in axial acquisition at 2.5$\mathrm{mm}$ slice thickness. Multiplanar reformatted images are used to better demonstrate bone anatomy and perform measurements. Patients are imaged in a supine position.

Normally the hard palate should be intact without any defect. In the case of cleft palate, there will be bony defect within the hard palate (Figure 1). In cases of Pierre Robin sequence, cleft palate is associated with micrognathia and glossoptosis and requires additional correction procedures such as mandibular distraction osteogenesis. ${ }^{6}$ Size, shape, and symmetry of the mandible can be well assessed. Length of the mandibular ramus can be

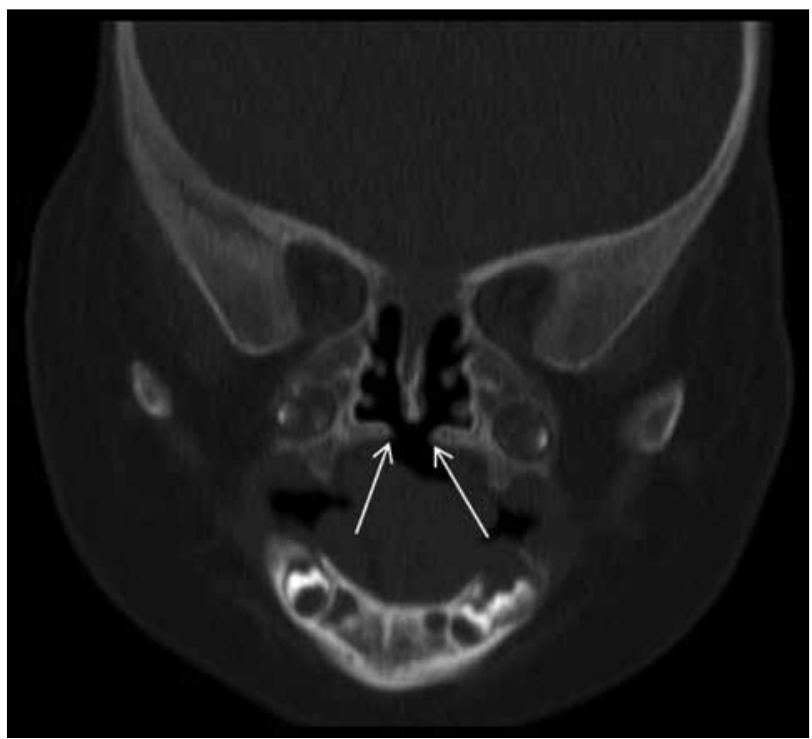

Figure 1. Bony cleft in hard palate. Coronal reformat of plain computed tomography images of the face and neck showing bony cleft in hard palate (arrows).

measured using sagittal oblique reformatted images (Figure 2). At least $17 \mathrm{~mm}$ length of the mandibular ramus is required to allow osteotomy and implant placement. ${ }^{6}$ Identification of the inferior alveolar nerve foramina is important to avoid nerve damage during osteotomy or pin insertion. ${ }^{6}$ The degree of airway obstruction can also be assessed (Figure 3).

\section{MAGNETIC RESONANCE IMAGING}

Our institution performs static MRI of the face and neck for patients with complicated cleft palate and those with primary correction failure. Our institution uses a 1.5-tesla MRI scanner (MAGNETOM Aera; Siemens, Erlangen, Germany) for all scans. A 20-channel cable-less Siemens head and neck coil are used during scanning. At the commencement of MRI study, we first acquire localiser images with fast gradient echo sequence within a few seconds. We then acquire T2-weighted images using turbo spin echo (TSE) technique. The sequences acquired include T2 3D SPACE (sampling perfection with application optimised contrasts using different flip angle evolutions) coronal, T2 TSE sagittal, T2 TSE coronal, and T2 TSE oblique coronal images. The T2 3D SPACE coronal sequence is performed first including the vertex of the skull and the entire mandible. With the help of this sequence, we select a plane parallel to the long axis of the levator veli palatini muscles, to obtain the T2 TSE oblique coronal images. This 


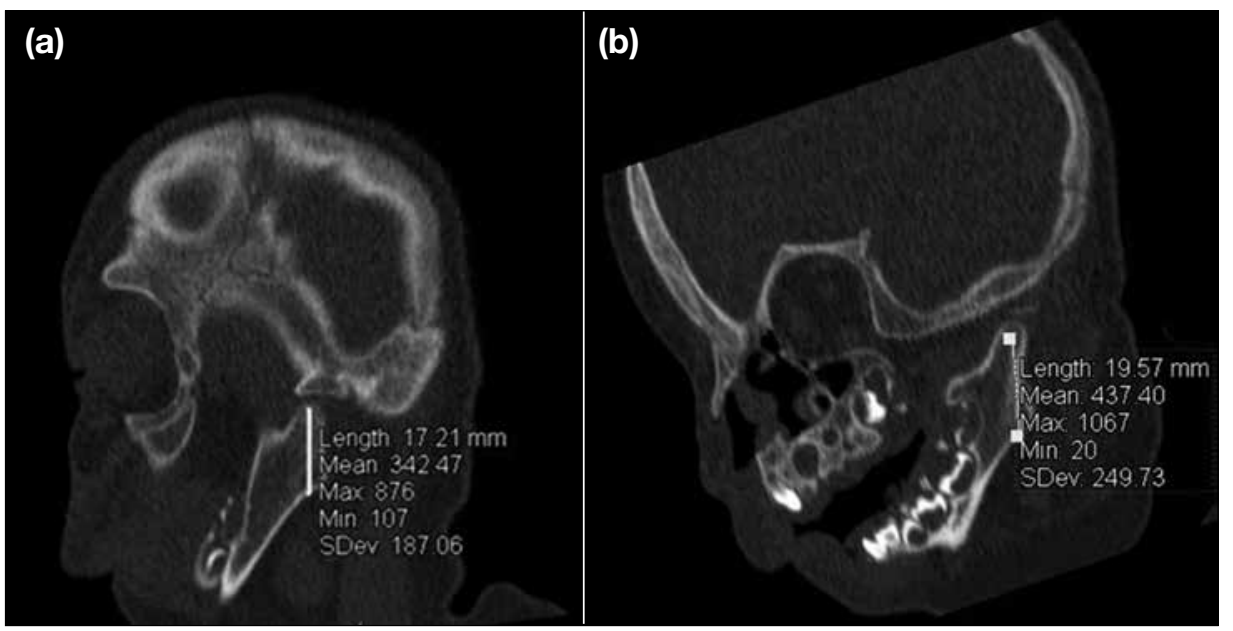

Figure 2. Oblique sagittal reformatted images of left (a) and right (b) mandibular rami. Mandibular length was measured from the antegonial notch to the junction of the neck of mandible and mandibular condyle. At least $17 \mathrm{~mm}$ length of the mandibular ramus is required to allow osteotomy and implant placement.

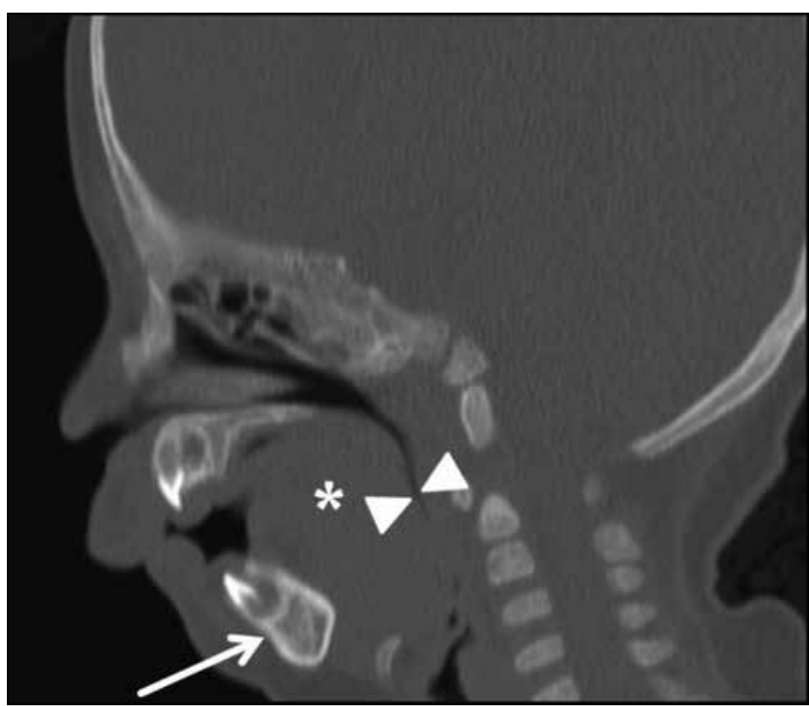

Figure 3. Sagittal reformatted computed tomography images of the face and neck showing retrognathia (arrow) and glossoptosis (asterisk) causing compression of the oropharyngeal airway (arrowheads).

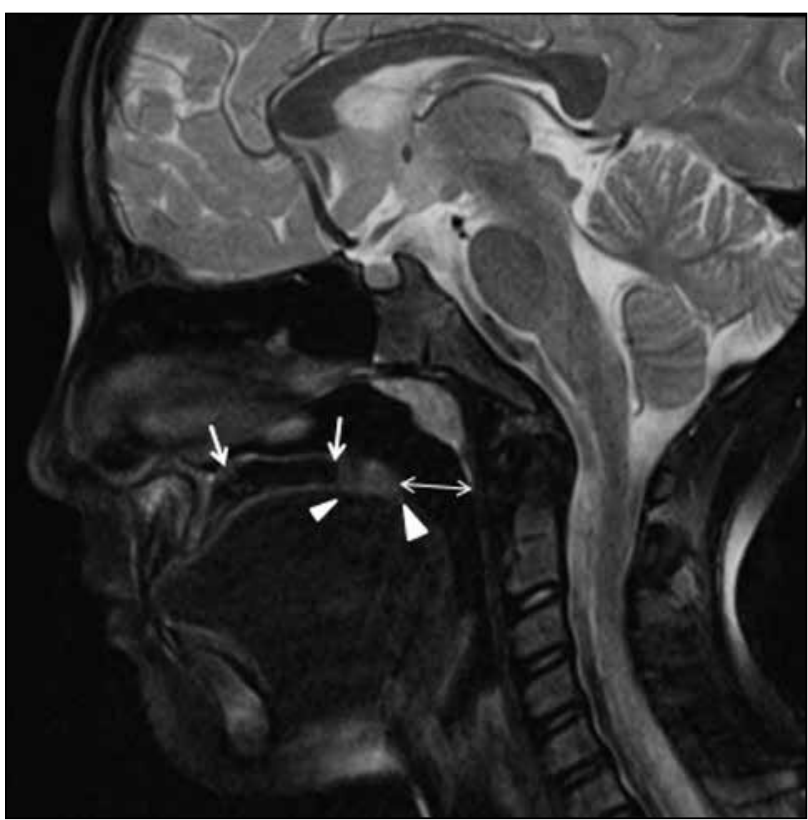

Figure 4. Magnetic resonance imaging T2-weighted midline sagittal image of the face and neck showing a shortened soft palate (arrowheads) with widened distance between the soft palate and posterior pharyngeal wall (double-headed arrow). The arrows indicate hypointense region corresponding to previous mucoperiosteal flap repair.

Table. Magnetic resonance imaging technical parameters for cleft palate.

\begin{tabular}{lccccccc}
\hline MRI sequence & $\begin{array}{c}\text { Field of view } \\
(\mathrm{mm})\end{array}$ & $\begin{array}{c}\text { Slice thickness } \\
(\mathrm{mm})\end{array}$ & Matrix size & $\begin{array}{c}\text { Pixel size } \\
(\mathrm{mm})\end{array}$ & $\begin{array}{c}\text { Repetition } \\
\text { time }(\mathrm{ms})\end{array}$ & $\begin{array}{c}\text { Echo time } \\
(\mathrm{ms})\end{array}$ & $\begin{array}{c}\text { Acquisition } \\
\text { time }(\mathrm{min}: \mathrm{s})\end{array}$ \\
\hline T2 3D SPACE coronal & $220 \times 165$ & 0.9 & $256 \times 256$ & 0.9 (isotropic) & 3200 & 379 & $4: 05$ \\
T2 TSE sagittal & $180 \times 180$ & 3 & $320 \times 320$ & $0.5 \times 0.7$ & 3580 & 90 & $3: 11$ \\
T2 TSE coronal & $180 \times 180$ & 3 & $320 \times 320$ & $0.5 \times 0.7$ & 2500 & 90 & $3: 02$ \\
T2 TSE oblique coronal & $200 \times 200$ & 3 & $384 \times 384$ & $0.5 \times 0.7$ & 2700 & 96 & $3: 00$ \\
\hline
\end{tabular}

Abbreviations: $3 \mathrm{D}$ = three-dimensional; $\mathrm{MRI}=$ magnetic resonance imaging; SPACE = sampling perfection with application optimised contrasts using different flip angle evolutions; TSE = turbo spin echo. 
specific oblique coronal plane allows better anatomical depiction of the levator veli palatini muscles. The T2 3D SPACE coronal sequence is also used to generate multiplanar reformatted images in the axial plane for anatomical correlation of velopharyngeal structures. The Table illustrates the detailed technical parameters. Only T2-weighted sequences without fat suppression are obtained as the presence of fat allows better depiction of various anatomical structures.

In patients with primary cleft palate and those with failed primary repair, the soft palate appears shortened with a widened distance between the soft palate and posterior pharyngeal wall (Figure 4). The levator veli palatini muscle sling appears thinned with loss of normal muscle signal in the midline, which is filled with fibrous tissue. The levator veli palatini muscle also lies along a relatively horizontal plane, leading to ineffective muscle contraction and velopharyngeal insufficiency (Figures 5 and 6).

Various measurements of velopharyngeal structures may be obtained, including hard palate length, velar length, velopharyngeal depth, palate width, and palate height (Figures 7 to 9). Previous studies have evaluated the range of values of velopharyngeal structures in normal subjects. ${ }^{4}$ Nonetheless, methods used to measure these parameters vary among studies, as do reference values and most include only a small number of patients. ${ }^{4} \mathrm{We}$ believe that providing the various measurements as
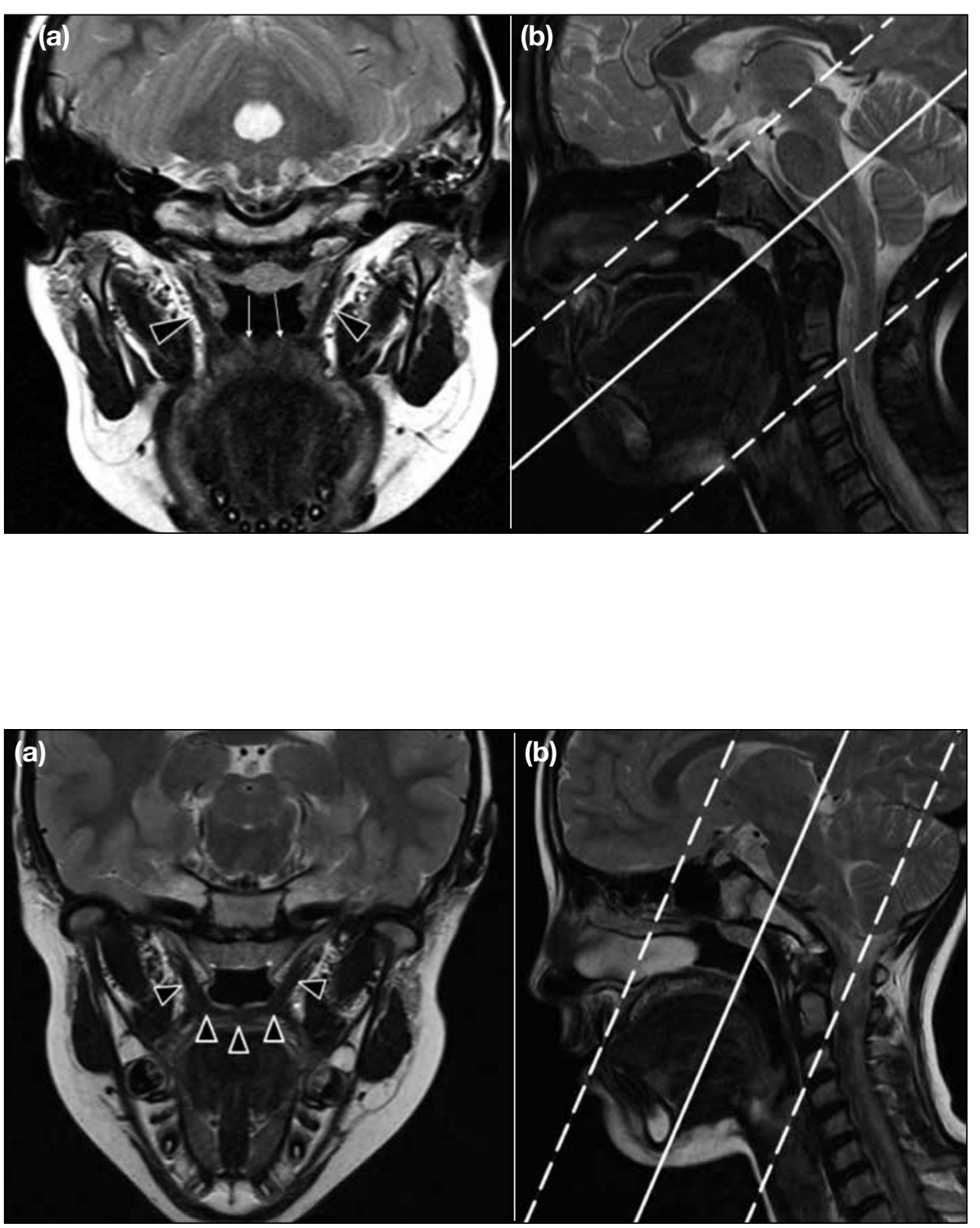

Figure 5. (a) Patient with failed primary cleft palate repair. Magnetic resonance imaging T2-weighted oblique coronal image showing thinning of the levator veli palatini muscle at the midline which is filled with fibrous tissue (arrows). Arrowheads indicate the remaining levator veli palatini muscle bulk. (b) Magnetic resonance imaging T2weighted sagittal image showing relative horizontal orientation of the levator veli palatini muscle sling (parallel dotted lines indicate the plane of the muscle sling; solid line indicates the image position of (a) which best shows the muscle sling).
Figure 6. (a) and (b) Same patient as Figure 5 who underwent palatoplasty with buccal myomucosal flap. (a) Magnetic resonance imaging T2weighted oblique coronal image showing muscle sling of the levator veli palatini appearing smooth with augmented muscle bulk (arrowheads indicate the levator veli palatini muscle sling after augmentation). No focal depression at the midline could be seen. (b) T2-weighted sagittal image showing more vertical orientation of the levator veli palatin muscle sling compared with Figure $5 b$ (parallel dotted lines indicate the plane of the muscle sling; solid line indicates the image position of Figure $6 a$ and best shows the muscle sling). 


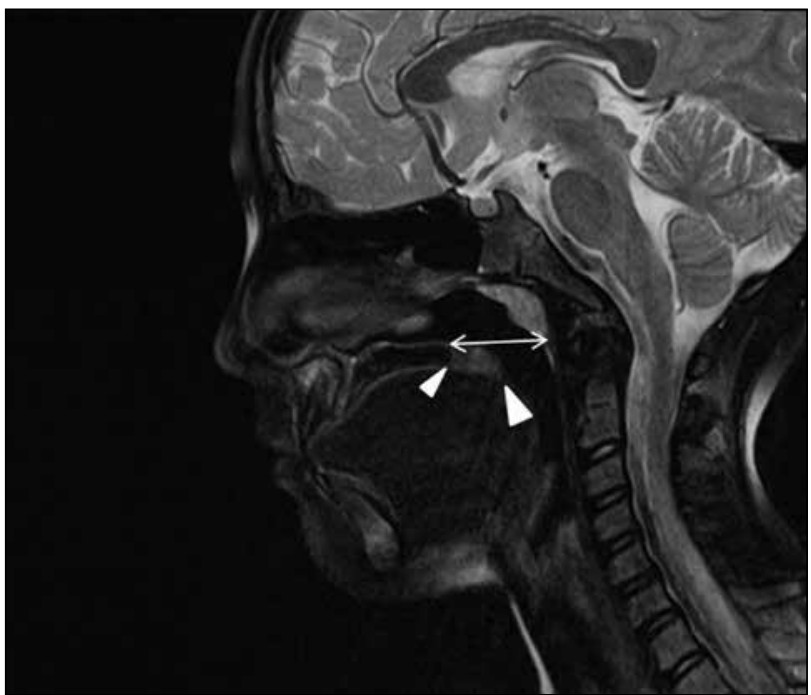

Figure 7. Magnetic resonance imaging T2-weighted midline sagittal image showing measurement of velar length (arrowheads) and velopharyngeal depth (double-headed arrow). Velar length is the distance between the posterior nasal spine (or posterior edge of mucoperiosteal flap for post-primary repair cases) and the tip of the velum. Velopharyngeal depth is the distance between the posterior nasal spine (or posterior edge of mucoperiosteal flap for post-primary repair cases) and the posterior pharyngeal wall along the plane of the hard palate. They are measured at the midline sagittal plane. a guide for surgeons is sufficient without the need to determine whether the different parameters follow a particular reference range. Surgical approaches include Furlow double-opposing Z-plasty, augmentation pharyngoplasty, pharyngeal flap surgery, and sphincter palatoplasty. ${ }^{7}$ According to Ruda et $\mathrm{al}^{7}{ }^{7}$ choice of treatment for velopharyngeal insufficiency depends on numerous factors. These include symmetry and degree of separation between the velum and pharynx, length of the palate and configuration of velopharyngeal muscles that can be assessed by MRI. A recent study by Ali et $\mathrm{al}^{5}$ demonstrated that static velopharyngeal measures on MRI in patients with cleft palate correlated with their speech performance. Velar length has been shown to have a strong positive linear correlation with degree of velar movement. ${ }^{5}$ Velar thickness has a significant moderate positive linear correlation with degree of velar movement and overall intelligibility of speech. ${ }^{5}$ The measurements provided in this pictorial essay help surgeons to select the most appropriate approach to optimise velar coverage in separating the nasopharynx and oropharynx during speech.
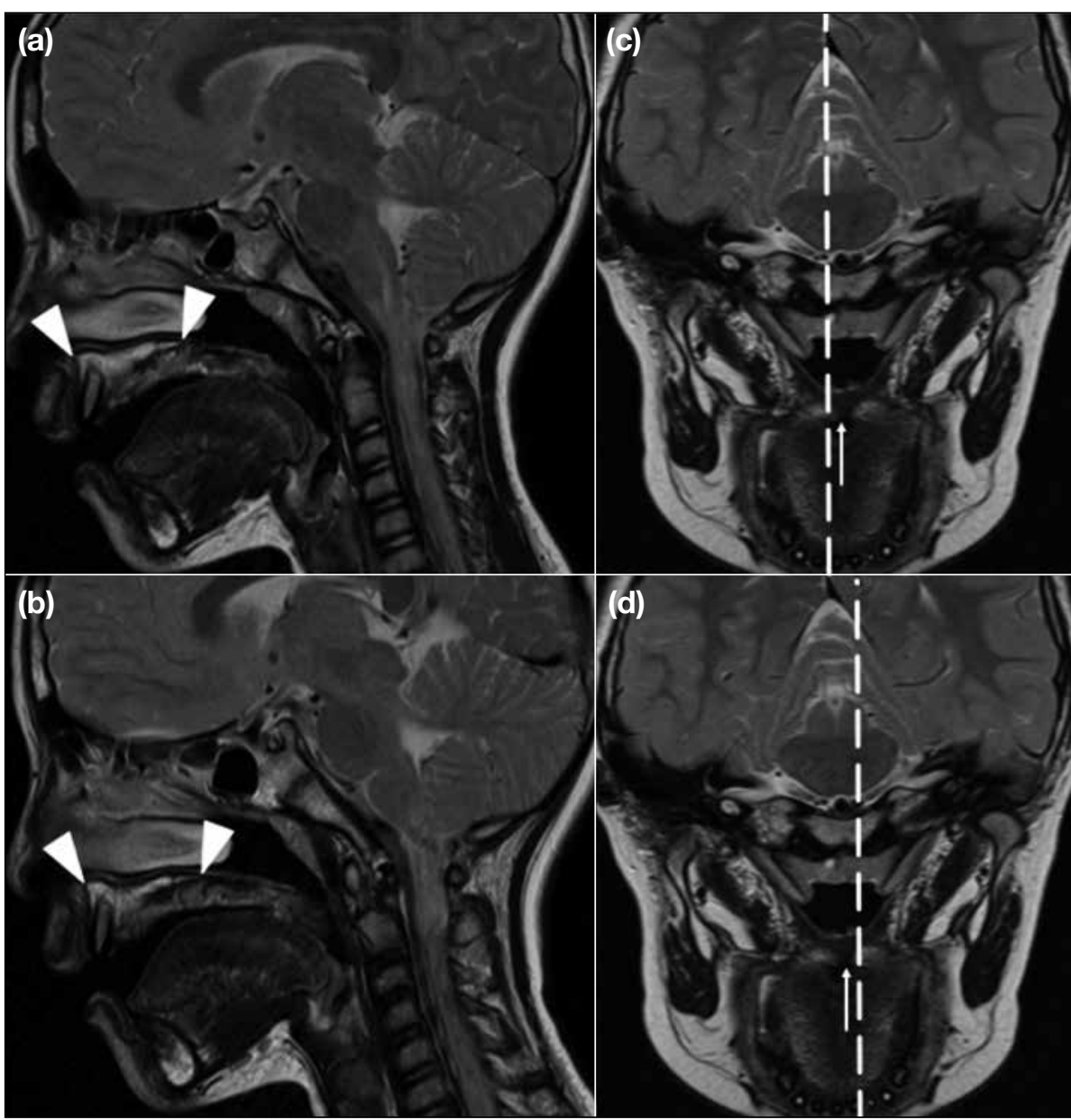

Figure 8. (a) and (b) Magnetic resonance imaging T2-weighted sagittal images obtained at right and left paramedian positions, respectively in a patient with bifid hard palate. Hard palate length was measured from the anterior to posterior nasal spine (arrowheads). (c) and (d) Magnetic resonance imaging T2-weighted coronal oblique images of the same patient. Dotted lines in (c) and (d) indicate the locations where (a) and (b) were obtained, respectively. White arrows indicate the midline bone cleft. 


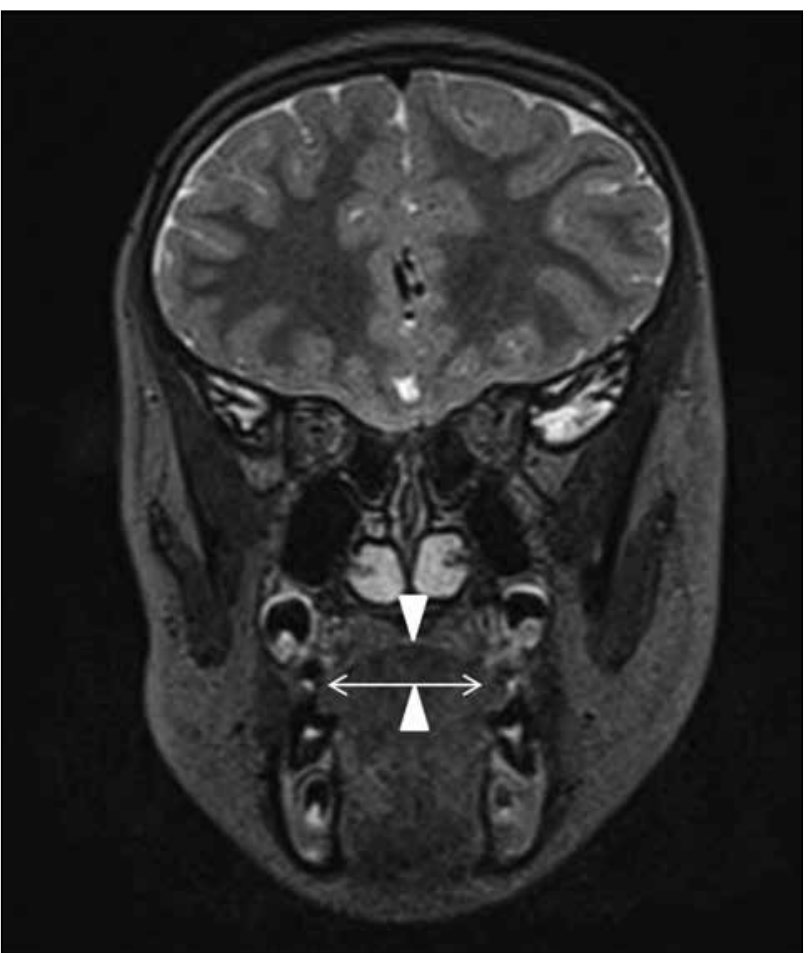

Figure 9. Magnetic resonance imaging T2-weighted coronal image illustrating palate width and height. True coronal plane was used for accurate measurement of palate width and height. Palate width (double-headed arrow) was measured at the plane of the second molar teeth between the free gingival lingual margins. Palate height (arrowheads) was measured using a line perpendicular to the palate width.

\section{CONCLUSION}

Preoperative imaging of the velopharyngeal apparatus is crucial to facilitate operative management. Crosssectional imaging allows non-invasive, accurate assessment of velopharyngeal and bone anatomy compared with other modalities. A multidisciplinary team that involves paediatric surgeons, maxillofacial surgeons, and radiologists is important for successful treatment of patients with cleft palate deformities.

\section{REFERENCES}

1. Choi WK. Primary cleft lip and palate repair. HK Med Diary. 2018;23:7-14.

2. United Christian Hospital, Hospital Authority, Hong Kong SAR Government. Cleft lip/cleft palate services. Hospital Authority, Hong Kong. Available from: http://www3 .ha.org.hk/uch/internet/ show.aspx?p=spCleft. Accessed 31 Jan 2019.

3. Abramson ZR, Peacock ZS, Cohen HL, Choudhri AF. Radiology of cleft lip and palate: imaging for the prenatal period and throughout life. Radiographics. 2015;35:2053-63.

4. Perry JL, Sutton BP, Kuehn DP, Gamage JK. Using MRI for assessing velopharyngeal structures and function. Cleft Palate Craniofac J. 2014;51:476-85.

5. Ali A, El Shamy M, AbdElmonem A, Hamza F, El Banoby T. Role of MRI in detection of repaired cleft palate muscles and correlation to speech. Egypt J Hosp Med. 2018;73:7604-9.

6. Meyers AB, Zei, MG, Denny AD. Imaging neonates and children with Pierre Robin sequence before and after mandibular distraction osteogenesis: what the craniofacial surgeon wants to know. Pediatr Radiol. 2015;45:1392-402.

7. Ruda JM, Krakovitz P, Rose AS. A review of the evaluation and management of velopharyngeal insufficiency in children. Otolaryngol Clin North Am. 2012;45:653-69, viii. 